\title{
Zur Diskussion
}

\section{Alexander Botte* \\ Empfohlene Schließung der ZB MED folgt nicht nachvollziehbarem Muster/Paradigma!}

DOI 10.1515/iwp-2016-0038

Der Senatsausschuss Evaluierung (SAE) der Leibniz-Gemeinschaft (WGL) hat mit der ZB MED (Leibniz Informationszentrum Lebenswissenschaften) ein weiteres Institut zur Schließung als eigenständige Einrichtung verurteilt, denn nichts anderes bedeutet die Empfehlung zur Beendigung der Förderung durch Bund und Länder im Rahmen der WGL. Wie schon im Fall des FIZ Chemie trifft es eine Fachinformationseinrichtung und erneut kann sich die Empfehlung in ihrer Radikalität nicht auf den zuvor eingereichten Bericht der Evaluierungskommission berufen, die keine Schließung nahelegte.

Mit dem Gesamturteil, dass es der ZB MED „nicht in dem notwendigen Maß gelungen ist, sich auf die erheblichen Veränderungen im Fachinformationswesen einzustellen“, sei vor dem Hintergrund, dass „das klassische Arbeitsfeld der ZB MED, das der Senat vor einigen Jahren noch als unverzichtbar für die bibliothekarische Versorgung in Deutschland ansah, ...kontinuierlich an Bedeutung" verliert, eine Weiterförderung nicht zu empfehlen.

Dabei war die ZB MED diese Herausforderung seit Jahren mit mehreren strategischen Maßnahmen angegangen: Sie verbreiterte mit der Ausdehnung auf die Lebenswissenschaften ihr disziplinäres Spektrum und ging mit Goportis eine multidisziplinäre Kooperation ein, die sowohl eine Produkt- wie eine Innovationsdimension hat. Sie setzte mit Open Access einen klaren und international relevanten Schwerpunkt, konnte mit dem Publikationsportal PUBLISSO auch eine vom Senat anerkannte Beachtung finden und bewährte sich in diesem Feld als starker nationaler und internationaler Player. Die Berufung einer W2-Professur für Informatik stand kurz bevor und eine weitere Berufung, mit der die neue Leitung des Instituts verbunden sein sollte, war in Vorbereitung. Gerade die Berufungen kommen aber für den Senat zu spät, der eine frühere gesamtkonzeptionelle Ausrichtung auf eine „for-

*Kontaktpersonen: Alexander Botte, Deutsches Institut für Internationale Pädagogische Forschung, Schloßstraße 29, 60486 Frankfurt am Main, E-Mail: botte@dipf.de schungsbasierte“ Weiterentwicklung der Infrastrukturen und erste Ergebnisse dazu einfordert:

„Es ist nicht gelungen, die Forschungsleistungen gegenüber der letzten Evaluierung signifikant auszuweiten und $\mathrm{zu}$ verbessern. Ein Forschungskonzept gibt es nach wie vor nicht. Der Senat beurteilt dies als nicht hinreichend [...]. Angesichts der geringen Forschungsleistungen konnte nicht erreicht werden, studentische Qualifikationsund Promotionsarbeiten in die Arbeiten der ZB MED systematisch zu integrieren, wie dies 2012 empfohlen worden war.“1

Auch wenn das Evaluationsgutachten insgesamt eine Reihe kritischer Punkte anmerkt, diese aber auch in einen sinnvollen Zeithorizont stellt, wird in der zusammenfassenden Bewertung des Senats deutlich, dass ein ganz zentraler Grund für die verweigerte weitere Förderung darin gesehen wird, dass die ,angewandte Forschung und Methodenentwicklung“„,hinter den Erfordernissen“ zurückbleibt. Seit knapp zehn Jahren - auch bei der Abwicklung des FIZ Chemie 2006 lassen sich entsprechende Formulierungen in der Stellungnahme des Senats identifizieren wird die spezifische Bewertung von Fachinformationseinrichtungen, die in ihrer ganzen Anlage, Tradition und personellen Aufstellung Dienstleistungseinrichtungen sind, allmählich aufgeweicht und das Kriterium der „Forschungsbasierung" rückt zunehmend in den Vordergrund.

Die WGL ist die einzige der fünf außeruniversitären Forschungsgemeinschaften, deren Mission explizit auch „wissenschaftliche Infrastrukturen“ und „forschungsbasierte Dienstleistungen“ einschließt und „Schwerpunkte im Wissenstransfer" setzt. Folglich sind die größten Fachinformationseinrichtungen Mitglied dieser - früher als „Blaue-Liste-Einrichtungen“ geführten - Wissenschaftsgemeinschaft. Obwohl dieses „Alleinstellungsmerkmal“ von der WGL stets hochgehalten wird, folgt der Katalog der Evaluierungskriterien aber weitgehend demjenigen, der für reine Forschungseinrichtungen gültig ist: Dies sind vor

1 http://www.leibniz-gemeinschaft.de/fileadmin/user_upload/dow nloads/Evaluierung/Senatsstellungnahmen/ZB_MED_-_Senatsstellu ngnahme_vom_17_03_2016_mit_Anlagen.pdf (S. 4). 
allem Forschungsdrittmittel, Anzahl an Peer-Review-Publikationen, Anzahl an Promotionen.

Es ist der WGL in den letzten Jahren trotz der Bemühungen des vor kurzem aufgelösten „Interdisziplinären Verbundes der Infrastruktureinrichtungen (IVI)“ nie hinreichend gelungen, angemessene Qualitätskriterien für Infrastruktureinrichtungen in die Evaluierung zu integrieren, wozu sicherlich auch die große Heterogenität der Infrastruktureinrichtungen innerhalb der WGL beigetragen hat. Letztlich haben die Evaluierungskommissionen dem in der Regel dadurch Rechnung getragen, dass sie von einer Infrastruktureinrichtung ein geringeres Level bei den „harten“ Forschungskriterien akzeptiert haben, ohne damit aber den spezifischen Leistungen einer Infrastruktureinrichtung durch quantifizierbare Indikatoren gerecht zu werden.

Auch Fachinformationseinrichtungen der WGL, die aufgrund ihrer multidisziplinären strategischen Bedeutung sich weiterhin einer spezifischeren Bewertung erfreuen können, werden durch ihre Evaluierungen massiv aufgefordert, „die Ausweitung der anwendungsorientierten Forschungs- und Entwicklungsarbeiten [...] unter Fokussierung auf klar definierte Themen fortzusetzen. Dies sollte auch eine deutliche Steigerung der Publikationstätigkeit erlauben." (TIB Hannover 2011)

Praktisch alle Fachinformationseinrichtungen der WGL kommen diesem Paradigmenwechsel inzwischen nach, indem sie die strategischen Leitungspositionen mit Professuren besetzen. Die ZBW hat als erste Bibliothekseinrichtung diesen Schritt bereits im Jahre 2010 mit einer Professur für Medieninformatik umsetzen können und damit eine strategische Entscheidung getroffen, die durch die damals sieben Jahre zurückliegende letzte Evaluierung in dieser Form noch gar nicht gefordert worden war. Die Neuausrichtung wurde bei der gleich anschließenden Evaluierung mit einem „Vorschuss“ an hoher Anerkennung für die Einsicht belohnt, ,[...] dass moderne Informationsdienstleistungen nur wettbewerbsfähig entwickelt werden können, wenn sie auf dem Fundament einer kontinuierlich betriebenen angewandten Forschung und Methodenentwicklung aufbauen." (ZBW 2011).

Aktuell scheint die Akzeptanz des neuen Forschungsprimats bei allen Fachinformationseinrichtungen hoch zu sein, eine Vielzahl von laufenden und abgeschlossenen Berufungsverfahren gemeinsam mit mehr oder weniger benachbarten Hochschulen belegt diesen Trend. Dabei wird außerhalb der betroffenen Institute häufig übersehen, dass die Umstellung auf umfassende Forschungsbasierung für diese Einrichtungen sehr aufwändig und grundsätzlich problematisch ist. Die Einrichtungen, die weiterhin ihre Dienstleistungen zu erbringen haben, müs- sen Anforderungen kombinieren, die nicht immer widerspruchsfrei erfüllt werden können: Letztlich geht es z.B. um die Frage, inwieweit etwa die Anzahl von Promotionen und nach engen disziplinären Standards abzufassenden Beiträgen in renommierten Fachzeitschriften wirklich zur Qualität von Infrastrukturdienstleistungen beitragen. Es kommt hier zu konkurrierenden Bedarfen des Mittel- und Personaleinsatzes.

Hinzu kommt, dass die flächendeckende Umstellung innovativer Maßnahmen auf eigene Forschung unter der Regie befristeter Drittmittelprojekte und Promotionsvorhaben erhebliche personelle und auch sachmittelbezogene Umstrukturierungsprozesse erfordert. Insbesondere $\mathrm{Bi}$ bliotheken sind hier benachteiligt, weil ihre traditionelle Personalstruktur dadurch geprägt ist, dass sie mit relativ geringem Anteil an wissenschaftlichem und IT-Personal ausgestattet sind. Dieses Fachpersonal ist dann im Wesentlich auch mit Daueraufgaben befasst, verfügt allerdings i.d.R. über die notwendige Erfahrung und Kompetenz, Innovationsprozesse zu initiieren und zu begleiten.

Gemeinsame Berufungen von außeruniversitären und hochschulischen Einrichtungen sind deutlich komplexer als gewöhnliche Berufungsverfahren, weil nicht nur unterschiedliche Anforderungsprofile (hier Instituts- bzw. Abteilungsleitung und Einbindung in die Mission einer außeruniversitären Forschungseinrichtung, dort hohe Anforderungen für Lehre und Forschung bei relativ starker inhaltlicher Autonomie des Stelleninhabers) in einer Person gebündelt werden müssen, sondern außerdem die Gewichtung dieser Anforderungen zwangsläufig noch kandidatenspezifisch ausgehandelt werden muss. Bei Einrichtungen, die neu in diese Form der Kooperation mit einer Hochschule einsteigen, ist i.d.R. in einem vorgeschalteten Prozess ein passender Kooperationsvertrag auszugestalten. All dies benötigt erfahrungsgemäß sehr viel Zeit, drei Jahre sind hier eine Erfahrungsgröße, die der ZB MED nicht mehr zugebilligt wurde. Die mit einer oder mehreren Professorenberufungen verbundene Neuorientierung wird darüber hinaus auch insgesamt verzögert, weil eine strategische Gesamtplanung vor Abschluss der Berufung(en) nur begrenzt einen Sinn macht.

Aber macht diese Anstrengung für alle Infrastrukturbereiche einer WGL-Einrichtung unter betriebswirtschaftlichen Aspekten überhaupt Sinn?

Fachinformationseinrichtungen und Bibliotheken nutzen aus guten Gründen traditionell Standardsoftware, soweit diese für ihre Produkte und Services zur Verfügung steht. Innovation im Infrastrukturbereich hat überwiegend eine zentrale technologische Komponente bzw. wird durch die zunehmende Digitalisierung von Infrastrukturdienstleistungen geprägt. Private Firmen und Hochschulen sind 
in diesem Feld starke Konkurrenz für eigenständige forschungsbasierte Optimierungen und meist besser auf die IT-intensiven und sehr langwierigen Innovationsprozesse ausgerichtet als Serviceeinrichtungen. Hochschulen und private Firmen finden i.d.R. auch bessere Voraussetzungen für FuE-Kooperationen vor, indem erstere den Forschungsanteil bestenfalls bis zur Entwicklung eines Prototyps und die privaten Firmen dann die Fortentwicklung bis zum marktfähigen Produkt übernehmen. Infrastruktureinrichtungen dagegen müssen sich am gesamten $\mathrm{FuE}$ Prozess beteiligen und diesen auch übergreifend steuern. Sie können und müssen dazu zwar auch kooperieren, tragen aber auch das gesamte Risiko, wenn z.B. eine Promotion kein nachnutzbares Ergebnis für die Praxis liefert.

Bei kühler Abwägung von materiellem und zeitlichem Aufwand dürfte in der Mehrzahl der Fälle die Innovation einer Infrastrukturkomponente günstiger $\mathrm{zu}$ realisieren sein, wenn sie angekauft und ggf. durch kleinere FuE-Aufwände an die disziplinären Gegebenheiten adaptiert wird. Aber das bringt nur sehr wenig Output im Bereich wissenschaftlicher Reputation und folglich für Professoren, die akademisch noch etwas werden wollen. Forschungsbasierte Infrastrukturen können also keine ubiquitäre Anforderung sein, sondern müssen fokussiert auf Felder angelegt sein, die sowohl wissenschaftlich als auch praktisch innovativ sind. Aktuelle Professorenberufungen an Fachinformationseinrichtungen zeigen eine starke Tendenz zur Informatik, die zwar auch praxisnahe Forschungsfelder kennt, aber in der wissenschaftsinternen Evaluierung häufig praxisferne Methoden bevorzugt. Für Fachinformationseinrichtungen ist eine informationswissenschaftliche
Expertise in besonderer Weise geeignet, weil sie Forschungs- und Anwendungsperspektiven besser verbindet.

Felder, auf denen innovative Infrastrukturen zu entwickeln sind, sind begrenzt. Es macht auch wenig Sinn, dass Innovationen von Infrastrukturen für jede Anwendungsdisziplin eigenständig entwickelt werden. Hier bieten sich von vornherein disziplinübergreifende Kooperationen an, die bei Bedarf spezifische Anpassungen an disziplinäre Besonderheiten ausdifferenzieren können. Entscheidendes Kriterium für die Sinnhaftigkeit solchen FuE-Engagements muss es aber sein, dass entsprechende Innovationen durch den „Markt“ nicht oder nicht hinreichend zur Verfügung gestellt werden. Für eine Infrastruktureinrichtung sollte immer die Optimierung der Gesamtdienstleistung im Vordergrund stehen, nicht die Generierung wissenschaftlicher Reputation über die bestmögliche Erfüllung entsprechender Leistungsindikatoren. Insbesondere kleinere Infrastruktur- und Fachinformationseinrichtungen geraten bei einer Umstellung auf forschungsbasierte Innovation schnell an Kapazitätsgrenzen, wenn diese über ein sachlich gebotenes Maß hinausgeht. Die gegenwärtige Evaluierungspraxis wird auf Dauer bestenfalls sehr große multidisziplinäre Infrastrukturen erhalten, wobei damit einer engen Abstimmung mit der Fachdisziplin, wie sie die DFG zurzeit für die Fachinformationsdienste $\mathrm{zu}$ einem zentralen Kriterium macht, entgegengewirkt wird. Die Wissenschaftspolitik und insbesondere die WGL müssen sich entscheiden, ob sie die Förderung von wissenschaftlicher Infrastruktur diesen Risiken durch eine forcierte Evaluation nach Forschungskriterien verstärkt aussetzen will. 